\title{
Analysis of the efficacy and prognostic factors of linear accelerator-based hypofractionated stereotactic radiotherapy for brain metastasis of non-small-cell lung cancer
}

Xi Wu ( 1632789113@qq.com )

Yunnan Cancer Hospital https://orcid.org/0000-0002-2802-5651

Yaoxiong Xia

Yunnan Cancer Hospital

Yu Hou

Yunnan Cancer Hospital

Lan Li

Yunnan Cancer Hospital

Li Wang

Yunnan Cancer Hospital

Rong Li

Yunnan Cancer Hospital

Fei Lu

Yunnan Cancer Hospital

Li Chang

Yunnan Cancer Hospital

Wenhui Li

Yunnan Cancer Hospital

\section{Research}

Keywords: hypofractionated stereotactic radiotherapy, brain metastases, non-small-cell lung cancer, linear accelerator

Posted Date: March 5th, 2020

DOl: https://doi.org/10.21203/rs.3.rs-16185/v1

License: (c) (1) This work is licensed under a Creative Commons Attribution 4.0 International License. Read Full License 


\section{Abstract}

Objective To investigate the efficacy and prognostic factors of treatment of brain metastases (BMs) of non-small-cell lung cancer (NSCLC) patients with hypofractionated stereotactic radiotherapy (HSRT) based on linear accelerators.

Methods A total of 80 NSCLC patients with BMs were treated with HSRT under the image guidance of tomotherapy (TOMO) or volumetric modulated arc therapy (VMAT) in Yunnan Cancer Hospital from Jan 1 st , 2017 to Aug 7 th , 2019. The outcome measures included overall survival (OS), intracranial local progression-free survival (iLPFS) and intracranial regional progression-free survival (iRPFS). The related prognostic factors were analyzed.

Results The median OS, iLPFS and iRPFS in 80 patients were 29.2 months, 19.3 months and 20.5 months, respectively. Using HSRT to treat BMs, whether combined with platinum-containing chemotherapy, targeted therapy, or both chemotherapy and targeted therapy, could improve OS ( $P$ $=0.027)$, iLPFS $(P=0.050)$ and iRPFS $(P=0.124)$. Among these patients, there were no significant differences in median OS (28.5 months vs 28.3 months, $P=0.785), 6$-month iLPFS rates $(81.3 \%$ vs $72.9 \%$, $P=0.998)$ and 6 -month iRPFS rates $(92.9 \%$ vs $74.8 \%, P=0.974)$ between patients with $4-10$ BMs and those with 1-3 BMs.

Conclusion The use of HSRT to treat BMs of NSCLC based on linear accelerators is safe and effective. Use of HSRT in combination with other antitumor therapies to treat BMs is a favorable prognostic factor that can affect OS and iLPFS. HSRT treatment of 4-10 BMs or 1-3 BMs resulted in similar OS, and there were no significant differences in 6-month iLPFS rates and 6-month iRPFS rates.

\section{Background}

Approximately $20 \%-65 \%$ of lung cancer patients experience brain metastases (BMs) at different stages of their disease. BMs occur in 30\%-50\% of non-small-cell lung cancer (NSCLC) patients and $10 \%-20 \%$ of patients present with BMs at first diagnosis, and this rate has increased significantly in recent years $(1,2)$. Patients with BMs have a poor prognosis and a serious decline in life quality. Local treatments such as surgery and radiotherapy can be used to control intracranial diseases more effectively on the basis of systemic therapy $(3,4)$.

Currently, BMs, including single and multiple BMs and postoperative tumor beds, are the most common indication of stereotactic radiosurgery (SRS). SRS can achieve 81-98\% local control in the treatment of BMs from lung cancer (5-7). Some studies compared single-fraction SRS (SF-SRS) and multifraction SRS (MF-SRS) for BMs, and the local control rate of MF-SRS was significantly better than that of SF-SRS, and the necrosis of the brain after radiotherapy was significantly lower than that of SF-SRS $(8,9)$. Due to the high risk of radioactive necrosis caused by the treatment of BMs in excessive doses of SF-SRS, the current trend is to use HSRT to control BMs as an alternative to SF-SRS (10). Although some studies have 
reported the efficacy of HSRT, there is no consensus on the recommended indications and dosages of HSRT (11-13).

The implementation of HSRT treatment for BMs is often carried out using gamma knives (GKS), and the application of a linear accelerator is even more common. This trial aims to explore the efficacy of using HSRT to treat BMs of NSCLC patients based on linear accelerators and to analyze the relevant prognostic factors.

\section{Materials And Methods}

\section{Study design and patient selection}

We reviewed NSCLC patients with BMs treated with HSRT under the image guidance of tomotherapy (TOMO) or volumetric modulated arc therapy (VMAT) in Yunnan Cancer Hospital from Jan $1^{\text {st }}, 2017$ to Aug $7^{\text {th }}, 2019$. Inclusion criteria: age $\geq 18$ years old, pathological diagnosis of NSCLC, MRI and/or CT exhibiting single or multiple brain lesions, number of BMs $\leq 10$, treatment of BMs with HSRT, complete medical records and follow-up data. Exclusion criteria: presence of other malignant tumors, HSRT not completed as planned, previous history of brain radiotherapy, extracranial death within 1 month after radiotherapy.

The outcome measures included overall survival (OS), intracranial local progression-free survival (iLPFS) and intracranial regional progression-free survival (iRPFS). OS was defined as the time from the discovery of BMs to death of any cause or final follow-up. The iLPFS was defined as the time between the end of radiotherapy for BMs and the first intracranial lesion or the last follow-up. The iRPFS was defined as the time between the end of radiotherapy for BMs and the appearance of new intracranial lesions or the last follow-up.

Relevant factors were collected: gender, age, Karnofsky Performance Scale (KPS) index, tumor site and type, time, site, size and number of BMs, extracranial metastasis and control, primary lesion status, data on recursive partitioning analysis (RPA), graded prognostic assessment (GPA), HSRT treatment of BMs in the same period as other antitumor treatment (surgery/chemotherapy/target/immunity, etc.), gene mutation and targeted therapy, time, method, dose and number of HSRT, follow-up treatment, disease progression time, method and follow-up treatment plan, etc. Ultimately, 80 patients were included in the analysis.

\section{Hypofractionated stereotactic radiotherapy}

All patients completed brain-enhanced MRI scans before radiotherapy and made head, neck and shoulder molds. The enhanced positioning CT scan was completed under the fixed head mold and body frame. The image fusion of enhanced MRI and enhanced localization CT was completed on the Pinnacle Planning System. The gross target volume (GTV) was delineated on the fused CT, and the planning target 
volume (PTV) was formed by $3 \mathrm{~mm}$ external expansion of GTV. Prescription dosages ensured at least $95 \%$ coverage of PTV and $100 \%$ coverage of GTV area.

The median PTV dose of 80 patients was $31.3 \mathrm{~Gy} / 3 \mathrm{f}$, and the dose range was $14-45 \mathrm{~Gy} / 2-9 \mathrm{f}(5 \mathrm{f} / \mathrm{w})$. Fifty-one patients $(63.8 \%$ ) received a dose of $30 \mathrm{~Gy} / 3 \mathrm{f}$ PTV. Five patients $(6.2 \%)$ received a radiotherapy dose less than PTV $30 \mathrm{~Gy} / 3 \mathrm{f}$ and a median dose of $20.6 \mathrm{~Gy} / 3 \mathrm{f}$ in the PTV range of 14-27 Gy/2-3 $\mathrm{f}$. Twenty-four patients $(30.0 \%$ ) received radiotherapy doses greater than PTV $30 \mathrm{~Gy} / 3 \mathrm{f}$, and PTV median dose of $36.4 \mathrm{~Gy} / 5 \mathrm{f}$, with a range of PTV $34-45 \mathrm{~Gy} / 3-9 \mathrm{f}$. For radiotherapy techniques, 37 patients (46.3\%) received TOMO, and 43 patients $(53.7 \%)$ received VMAT.

All patients underwent clinical follow-up, including a craniofacial MRI or enhanced CT scan 1 month after the end of radiotherapy and every 3 months thereafter.

\section{Statistical analysis}

Statistical analysis was carried out using SPSS 22.0 software. Survival analysis was performed with Kaplan-Meier method. The log-rank test was used for single variable analysis of prognostic factors. The variables with $P<0.1$ in the single variable analysis are included in the Cox scale risk regression model for multivariate analysis to evaluate the independent prognostic factors associated with OS, iLPFS, and iRPFS. $P<0.05$ was statistically significant.

\section{Results}

\section{Baseline characteristics of patients}

Among the 80 patients included in our study, 36 were female (45.0\%) and 44 were male (55.0\%), with an age distribution ranging from 34 to 76 years old and a median age of 54 years old. Twelve patients $(15.0 \%)$ had KPS $\leq 70$ points. BMs were found in 29 patients $(36.3 \%)$ at initial diagnosis. The number of BMs in 65 patients $(81.3 \%)$ was no more than 3 , and the maximum diameter of BMs in 68 patients (85.0\%) was no more than $3 \mathrm{~cm}$. There were 63 patients (78.8\%) with a pathological type of adenocarcinoma, 16 patients (20.0\%) with clear EGFR mutations, 3 patients (3.8\%) with ALK mutations, 2 patients $(2.5 \%)$ with ROS-1 mutations, 1 patient (1.3\%) with KRAS mutation, 32 patients (40.0\%) wild type for the tested genes, and 26 patients (32.5\%) with no genetic testing. In the same period, 46 patients (57.5\%) received other antitumor therapy, including 18 patients $(22.5 \%)$ who received platinum-based chemotherapy, 16 patients $(20.0 \%)$ who received targeted therapy, 12 patients $(15.0 \%)$ who were treated with platinum-containing chemotherapy combined with targeted therapy. Of the 28 patients $(35.0 \%)$ who received targeted therapy, 14 patients $(17.5 \%)$ received EGFR-TKI targeted therapy, 5 patients $(6.3 \%)$ received ALK-TKI targeted therapy, and 9 patients $(11.3 \%)$ received vascular targeted therapy (Table 1$)$.

\section{Survival and intracranial control}


In our study, the median follow-up period was 22.4 months, ranging from 1-33 months to the last followup date of October $20^{\text {th }}, 2019$, in which 23 patients $(28.8 \%$ ) died and 31 patients $(38.8 \%)$ had progression of intracranial lesions. Twenty-two patients $(27.5 \%)$ were actively treated for intracranial progression.

\section{Overall survival}

The median OS of patients in our study was 29.2 months, ranging from 2 to 43 months, and the OS rates at $6,12,24$ and 36 months were $89.1 \%, 79.0 \%, 63.1 \%$ and $51.0 \%$, respectively (Figure 1 ). Single-factor analysis showed that the KPS score $(P=0.001)$, pathological type $(P<0.001)$, RPA grade $(P=0.007)$, extracranial metastasis status $(P=0.012)$, number of extracranial metastasis organs $(P=0.042)$, and whether the extracranial and primary lesions were stable $(P<0.001)$ were prognostic factors for $0 S$.

The median OS from HSRT combined with other antitumor therapies reached 31.6 months, but the difference was not statistically significant compared to 22.7 months from BMs treated with HSRT alone $(P=0.094)$. Our study suggested that there was no significant difference in median OS between patients with 4-10 BMs and 1-3 BMs treated with HSRT (28.5 months vs 28.3 months, $P=0.785$ ).

The multivariate analysis showed that the independent adverse prognostic factors affecting OS included KPS $<70$ (HR: $0.188,95 \% \mathrm{Cl}: 0.044-0.807, P=0.024)$, non-adenocarcinoma (HR: $12.315,95 \% \mathrm{Cl}: 2.429-$ $62.423, P=0.002$ ), other mutations of non-EGFR mutations (HR: $12.423,95 \% \mathrm{Cl}: 1.084-142.297, P=0.045$ ) and HSRT treatment without other antitumor treatment during the same period (HR: $0.108,95 \% \mathrm{Cl}: 0.024-$ $0.495, P=0.004)$ (Figure 1) (Supplementary Table 1).

\section{The intracranial local progression-free survival time}

The median iLPFS of patients in our study was 19.3 months, ranging from 1 to 32 months. The iLPFS rates at $6,12,24$ and 30 months were $78.3 \%, 63.9 \%, 36.5 \%$ and $36.5 \%$, respectively (Figure 2 ). The singlefactor analysis showed that KPS>70 $(P=0.006)$, adenocarcinoma $(P=0.001)$, other antitumor treatments received during the same period of HSRT $(P=0.033)$, a grade of RPA I $(P=0.010)$, no metastasis of extracranial organs $(P=0.044)$ and the stability of extracranial and primary lesions $(P<0.001)$ were favorable prognostic factors of iLPFS.

The median iLPFS of patients who received HSRT combined with other antitumor therapies reached 22.0 months, and the difference was statistically significant compared to 12.0 months in patients whose BMs were treated with HSRT alone $(P=0.033)$. Our study suggested that there was no significant difference in 6-month iLPFS rates between patients with 4-10 BMs and 1-3 BMs treated with HSRT (81.3\% vs $72.9 \%$, $P=0.998)$.

The multivariate analysis showed that the independent adverse prognostic factors of iLPFS included nonadenocarcinoma (HR: 4.952, 95\% Cl: 1.074-22.832, $P=0.040$ ), other mutations of non-EGFR mutations (HR: 7.715, 95\% Cl: 1.059-56.230, $P=0.030$ ) and HSRT treatment of BMs did not receive other antitumor 
treatment during the same period (HR囚0.124,95\%Cl『0.029-0.536هP=0.005)(Figure 2)(Supplementary Table 2).

\section{The intracranial regional progression-free survival time}

The median iRPFS of patients in our study was 20.5 months, ranging from 1 to 32 months. The iRPFS rates at $6,12,24$ and 30 months were $78.0 \%, 67.4 \%, 47.5 \%$ and $47.5 \%$, respectively (Figure 3 ). The singlefactor analysis showed that KPS $>70(P=0.005)$, adenocarcinoma $(P<0.001)$, RPA I $(P=0.007)$, no metastasis of extracranial organs and stability of extracranial and primary lesions $(P<0.001)$ were the favorable prognostic factors of iRPFS.

The median iRPFS of patients who received HSRT combined with other antitumor therapies reached 22.7 months, and the difference was not statistically significant compared to 16.5 months of patients received HSRT alone ( $P=0.097)$. Our study suggested that there was no significant difference in 6-month iRPFS rates between patients with 4-10 BMs and 1-3 BMs treated with HSRT ( $92.9 \%$ vs $74.8 \%, P=0.974)$.

The multivariate analysis showed that the independent adverse prognostic factors of iRPFS included non-adenocarcinoma (HR: 4.765, 95\%Cl: 1.093-20.781, $P=0.038$ ), and HSRT treatment of BMs did not receive other antitumor treatment during the same period (HR: $0.160,95 \% \mathrm{Cl}: 0.033-0.772, P=0.023$ ) (Figure 3) (Supplementary Table 3).

\section{Secondary analysis of other antitumor therapies}

The median OS of HSRT combined chemotherapy for the same period was 30.1 months, the combined target was 31.1 months, and both combined chemotherapy and target was 27.9 months, which better than the 22.7 months of BMs treated with HSRT alone. The median iLPFS of HSRT combined chemotherapy for the same period was 15.6 months, the combined target was 22.1 months, and both combined chemotherapy and target was 20.8 months, which better than the 12.0 months of BMs treated with HSRT alone. The median iRPFS of HSRT combined chemotherapy for the same period was 17.4 months, the combined target was 22.1 months, and both combined chemotherapy and target was 19.9 months, which better than the 16.5 months of BMs treated with HSRT alone.

Above all, our study suggested that compared to patients who did not receive other antitumor treatments during the same period of HSRT, whether combined with platinum-containing chemotherapy, targeted therapy, or combined chemotherapy and targeted therapy could improve OS, iLPFS, and iRPFS.

The specific antitumor therapy received in the same period of HSRT treatment for BMs was included in the multifactor analysis of OS, iLPFS and iRPFS. The multifactor analysis showed that compared to HSRT of BMs without receiving other antitumor treatments, whether combined with platinum-containing chemotherapy, targeted therapy, or combined chemotherapy and targeted therapy could improve OS $(P=0.027)$, iLPFS $(P=0.050)$ and iRPFS $(P=0.124)$ (Figure 4). The differences in OS and iLPFS were statistically significant, but there was no significant difference in iRPFS (Supplementary Table 4-6). 


\section{Toxicity}

A total of nine patients $(11.3 \%)$ in our study had physical sensation and movement disorders, and 34 patients (42.5\%) had clear neurological symptoms, such as dizziness, headache and other symptoms, and had different degrees of symptom relief after completion of cerebral HSRT. Three patients (3.8\%) had seizures during HSRT, which were controlled after anti-epileptic treatment, and one patient (1.3\%) had occasional seizures after radiotherapy. Fourteen patients $(17.5 \%)$ had mild to moderate consciousness disturbance. Four patients (5.0\%) had grade II-III myelosuppression. Seventeen patients $(21.3 \%)$ had mild to moderate gastrointestinal reactions. One patient (1.3\%) developed liver function impairment. In our study, CT and MRI examination did not indicate any obvious cerebral radionecrosis.

\section{Discussion}

SRS is becoming a major force in the treatment of BMs. It can be used for single or multiple BMs, and the median OS can be up to 20 months (14-16). It can be used as adjuvant therapy after surgery for BMs, with a median OS up to 12.2 months (17). SRS can be applied to re-SRS that has previously received SRS treatment for more than 6 months and confirming tumor recurrence, and the second SRS median OS could be up to 11 months $(18,19)$. It can be used for rescue treatment after whole brain radiotherapy (WBRT)failure, delay or avoid WBRT (20-22). In our study, HSRT was used to treat 80 patients with NSCLC BMs, with a median OS of 29.2 months, a median iLPFS of 19.3 months, a median iRPFS of 20.5 months, and a lower incidence of toxic reactions, which was better than the results of most previous studies.

In a retrospective trial, analyzed 2,966 patients who received a single SRS treatment for BMs (not limited to primary tumors), and statistical analysis suggested that patients with KPS $\geq 80$, primary cancer under control, and no extracranial metastasis may have longer OS and a higher risk of radioactive complications (23). In our study, a multifactor analysis suggested that KPS $<70$ is an independent adverse prognosis factor affecting OS $(P=0.024)$. It did not suggest that no extracranial metastasis and the control of extracranial and primary cancer were the independent prognostic factors affecting OS, iLPFS and iRPFS. Rosell et al.(24) showed that adenocarcinoma had a longer survival period than squamous cell carcinoma ( 8.8 months vs 5.4 months, $P=0.01$ ). Our study suggested that nonadenocarcinoma is an independent adverse prognostic factor affecting OS, iLPFS, and iRPFS $(P<0.05)$, consistent with previous studies. GPA and RPA can predict the survival of patients with BMs (25-27). In our study, the single-factor analysis suggested that GPA had some effect on OS and iRPFS $(P<0.1)$, and RPA had some effect on OS, iLPFS and iRPFS $(P<0.05)$. However, the multivariate analysis did not suggest that GPA and RPA were the independent prognostic factors affecting OS, iLPFS and iRPFS.

At present, the main treatment for BMs of NSCLC include the combination or nonunion of local therapy and systemic therapy. Several studies have shown that intracranial tumor remission rates can be improved by combined/noncombined platinum-based chemotherapy (whether sequential or synchronous) in patients with BMs of NSCLC, but there was no significant difference between PFS and 
OS, and radiotherapy combined chemotherapy increased the incidence of adverse reactions $(28,29)$. BM is more likely in NSCLC patients with positive driver genes, with a rate of $60 \% \mathrm{BM}$ in patients with EGFR mutation and an incidence of BM of approximately $45 \%-70 \%$ in ALK-positive patients $(30,31)$. The discovery of driver genes such as EGFR, ALK, ROS1 and the emergence of related targets provide a more simple and effective treatment for patients with BMs, further prolonging survival. The combination of EGFR-TKI and radiotherapy for BMs can improve Os and PFS, but the combination mode of EGFR-TKI and radiotherapy has not been determined. EGFR status was differentiated in only a few clinical studies of EGFR-TKI concurrent radiotherapy for BMs, and the results varied. A Small Phase II study suggested that in EGFR mutated patients with BMs of NSCLC, concurrent EGFR-TKI and radiotherapy have a slight OS and PFS benefit compared to radiotherapy alone (22.0 months vs 7.5 months) (32). Chang W et al. (33) retrospectively analyzed 351 newly diagnosed EGFR mutant lung adenocarcinoma BMs patients who were not subjected to targeted therapy and studied the sequence of EGFR-TKI and radiation therapy for BMs. The results suggested that patients with the SRS sequence EGFR-TKI had longer OS (45 months vs 25 months, $P<0.001$ ) and that delayed radiotherapy reduced OS. The BRAIN study suggested that for patients with EGFR mutations, iPFS improved when EGFR-TKI used prior to BMs radiotherapy $(10.0$ months vs 4.8 months, $P=0.014)(34)$. For patients with NSCLC BMs in ALK-positive or ROS1-positive, crizotinib is widely used in clinical practice (35-37). Vascular-targeted therapy (e.g., bevacizumab, etc.) is also widely studied in patients with NSCLC BMs $(38,39)$.

In our study, the multivariate analysis suggested that other mutations of non-EGFR mutations was independent prognostic factor affecting OS $(P=0.045)$ and iLPFS $(P=0.030)$. We considered whether this was because there were fewer patients $(7.5 \%)$ with non-EGFR mutations in our study: three patients (3.8\%) had ALK-positive mutations, two patients (2.5\%) had ROS1-positive mutations, and one patient $(1.3 \%)$ had KRAS mutations. Patients that were ALK-positive and ROS1-positive were treated with crizotinib, which was unevenly distributed across the groups, resulting in a large difference in prognosis. Of the 28 patients $(35.0 \%)$ in our study, 14 patients (17.5\%) received EGFR-TKI targeted therapy, 5 patients (6.3\%) received ALK-TKI targeted therapy, and 9 patients (11.3\%) received vascular targeted therapy; there were no significant differences in OS $(P=0.597)$, iLPFS $(P=0.711)$ and iRPFS $(P=0.670)$ in the univariate analysis. The multifactorial analysis was not included because of the small number of cases and uneven stratification.

For patients with NSCLC BMs without a driver gene mutation, systemic chemotherapy is still the main method of therapy. The study on the combination of brain radiation and targeted therapy in driver genepositive NSCLC patients with BMs is mostly retrospective, with small sample sizes and inconsistent results. WBRT was the most common choice in the current study of combined chemotherapy/targeted therapy and radiotherapy for patients with BMs from NSCLC. There is a lack of research on HSRT combined with chemotherapy and targeted therapy for BMs. Our study suggests that, compared to patients who did not receive other antitumor treatments during the same period of HSRT, a combination with platinum-containing chemotherapy, targeted therapy, or both chemotherapy and targeted therapy could improve OS $(P=0.027)$, iLPFS $(P=0.050)$ and iRPFS $(P=0.124)$, but the best combination should be determined by large-scale prospective studies. 
In a prospective trial SRS-JLGK 0901, 1,194 patients with newly diagnosed BMs were recruited, and all patients were treated with SRS for BMs. The median OS of patients with $1 \mathrm{BM}(\mathrm{N}=455)$ was 13.9 months. The median OS of patients with $5-10 \mathrm{BMs}(\mathrm{N}=531)$ was not significantly different compared with the OS of patients with $2-4 \mathrm{BMs}(\mathrm{N}=208)$, at 10.8 months $(P<0.0001)$. There was no significant difference in the incidence of adverse events between the two groups $(P=0.89)(40)$. This experiment shows that SRS of 1-3 BMs or multiple BMs results in similar OS and similar toxicity and side effects.

In our study, when HSRT was used to treat 4-10 BMs compared with 1-3 BMs, and there was no significant difference in median OS ( 28.5 months vs 28.3 months, $P=0.785)$, 6-month iLPFS rates ( $81.3 \%$ vs $72.9 \%, P=0.998)$ and 6 -month iRPFS rates $(92.9 \%$ vs $74.8 \%, P=0.974)$, consistent with other studies. The median OS of patients with 4-10 BMs was slightly higher than that of patients with 1-3 BMs in our study, and the 6-month iLPFS rates and 6-month iRPFS rates were higher than those of patients with 1-3 BMs, which may be due to the large baseline difference between the two groups, with the majority (65.0\%) having 1-3 BMs and only a small minority (15.0\%) having 4-10 BMs.

\section{Limitations}

There are many limitations to our study. These include: the limiting factors inherent in retrospective studies, the small sample size, the imbalance of the baseline of patients after stratification in the group, the short follow-up period, the difficulty in assessing the associated nervous system side effects after brain radiotherapy and the complexity of HSRT doses. Therefore, the results of our study need to be carefully interpreted, and large-scale prospective studies are required to verify the credibility of the conclusions.

\section{Conclusion}

The use of HSRT to treat BMs of NSCLC based on linear accelerators is safe and effective. Use of HSRT in combination with other antitumor therapies to treat BMs is a favorable prognostic factor that can affect OS and iLPFS, whether combined with platinum-containing chemotherapy, targeted therapy, or both chemotherapy and targeted therapy. HSRT treatment of 4-10 BMs or 1-3 BMs resulted in similar OS, and there were no significant differences in 6-month iLPFS rates and 6-month iRPFS rates. KPS $<70$ is an independent adverse prognostic factor for OS. The non-adenocarcinoma subtype is an independent adverse prognostic factor for OS, iLPFS and iRPFS.

\section{List Of Abbreviations}




\begin{tabular}{ll}
\hline AbbreviationsFull name \\
\hline BMs & Brain metastases \\
NSCLC & Non-small-cell lung cancer \\
\hline HSRT & Hypofractionated stereotactic radiotherapy \\
\hline TOMO & Tomotherapy \\
\hline VMAT & Volumetric modulated arc therapy \\
\hline OS & Overall survival \\
\hline iLPFS & Intracranial local progression-free survival \\
\hline iRPFS & Intracranial regional progression-free survival \\
\hline SRS & Stereotactic radiosurgery \\
\hline SF-SRS & Single-fraction SRS \\
\hline MF-SRS & Multifraction SRS \\
\hline GKS & Gamma knives \\
\hline KPS & Karnofsky Performance Scale \\
\hline RPA & Recursive partitioning analysis \\
\hline GPA & Graded prognostic assessment \\
GTV & Gross target volume \\
PTV & Planning target volume \\
\hline WBRT & Whole brain radiotherapy \\
\hline
\end{tabular}

\section{Declarations}

\section{Ethics approval and consent to participate}

This study was approved by the Ethics Committee of the Third Affiliated Hospital of Kunming Medical University. Informed consent was waived by the committee because of the retrospective nature of this study. This trial was conducted in accordance with the Declaration of Helsinki. We confirm that patient data confidentiality was maintained.

\section{Patient consent for publication}

Not applicable.

\section{Availability of data and materials}

The datasets used and analysed during the current study are available from the corresponding author on reasonable request.

\section{Competing interests}

The authors declare that they have no competing interests.

\section{Funding}

Not applicable. 


\section{Authors' contributions}

$\mathrm{YX}, \mathrm{YH}, \mathrm{WL}$ design of the work. RL, FL, XW the acquisition, analysis. XW, LC, LW, LL interpretation of data, have drafted the work or substantively revised it, have approved the submitted version, have agreed both to be personally accountable for the author's own contributions and to ensure that questions related to the accuracy or integrity of any part of the work.

\section{Acknowledgements}

This work was supported by the National Natural Science Foundation of China (Nos.81660504, 81860536, 81560488 ), Yunnan Provincial Training Special Funds for High-level Health Technical Personnel (Nos.L-2018001, H-201624), Yunnan Health Science Foundation (Nos.2017NS191, 2018NS0065, 2018NS0064).

\section{References}

1. Mulvenna P, Nankivell M, Barton R, Faivre-Finn C, Wilson P, McColl E, et al. Dexamethasone and supportive care with or without whole brain radiotherapy in treating patients with non-small cell lung cancer with brain metastases unsuitable for resection or stereotactic radiotherapy (QUARTZ): results from a phase 3, non-inferiority, randomised trial. Lancet. 2016;388(10055):2004-14.

2. Waqar SN, Samson PP, Robinson CG, Bradley J, Devarakonda S, Du L, et al. Non-small-cell Lung Cancer With Brain Metastasis at Presentation. Clin Lung Cancer. 2018;19(4):e373-e9.

3. Patla A, Walasek T, Jakubowicz J, Blecharz P, Mitus JW, Mucha-Malecka A, et al. Methods and results of locoregional treatment of brain metastases in patients with non-small cell lung cancer. Contemp Oncol (Pozn). 2016;20(5):358-64.

4. Wang W, Song Z, Zhang Y. Efficacy of brain radiotherapy plus EGFR-TKI for EGFR-mutated non-small cell lung cancer patients who develop brain metastasis. Arch Med Sci. 2018;14(6):1298-307.

5. Aoyama H, Shirato H, Tago M, Nakagawa K, Toyoda T, Hatano K, et al. Stereotactic radiosurgery plus whole-brain radiation therapy vs stereotactic radiosurgery alone for treatment of brain metastases: a randomized controlled trial. JAMA. 2006;295(21):2483-91.

6. Chang EL, Wefel JS, Hess KR, Allen PK, Lang FF, Kornguth DG, et al. Neurocognition in patients with brain metastases treated with radiosurgery or radiosurgery plus whole-brain irradiation: a randomised controlled trial. Lancet Oncol. 2009;10(11):1037-44.

7. Kocher M, Soffietti R, Abacioglu U, Villa S, Fauchon F, Baumert BG, et al. Adjuvant whole-brain radiotherapy versus observation after radiosurgery or surgical resection of one to three cerebral metastases: results of the EORTC 22952-26001 study. J Clin Oncol. 2011;29(2):134-41.

8. Minniti G, Scaringi C, Paolini S, Lanzetta G, Romano A, Cicone F, et al. Single-Fraction Versus Multifraction (3 $\times 9$ Gy) Stereotactic Radiosurgery for Large $(>2 \mathrm{~cm}$ ) Brain Metastases: A Comparative Analysis of Local Control and Risk of Radiation-Induced Brain Necrosis. Int J Radiat Oncol Biol Phys. 2016;95(4):1142-8. 
9. Park K, Kim JW, Chung HT, Paek SH, Kim DG. Single-Session versus Multisession Gamma Knife Radiosurgery for Large Brain Metastases from Non-Small Cell Lung Cancer: A Retrospective Analysis. Stereotact Funct Neurosurg. 2019;97(2):94-100.

10. Ahmed KA, Sarangkasiri S, Chinnaiyan P, Sahebjam S, Yu HH, Etame AB, et al. Outcomes Following Hypofractionated Stereotactic Radiotherapy in the Management of Brain Metastases. Am J Clin Oncol. 2016;39(4):379-83.

11. Croker J, Chua B, Bernard A, Allon M, Foote M. Treatment of brain oligometastases with hypofractionated stereotactic radiotherapy utilising volumetric modulated arc therapy. Clin Exp Metastasis. 2016;33(2):125-32.

12. Masucci GL. Hypofractionated Radiation Therapy for Large Brain Metastases. Front Oncol. 2018;8:379.

13. Ruschin M, Sahgal A, Soliman H, Myrehaug S, Tsao M, Yeboah C, et al. Investigation of irradiated volume in linac-based brain hypo-fractionated stereotactic radiotherapy. Radiat Oncol. 2017;12(1):117.

14. Churilla TM, Chowdhury IH, Handorf E, Collette L, Collette S, Dong Y, et al. Comparison of Local Control of Brain Metastases With Stereotactic Radiosurgery vs Surgical Resection: A Secondary Analysis of a Randomized Clinical Trial. JAMA Oncol. 2019;5(2):243-7.

15. Shinde A, Akhavan D, Sedrak M, Glaser S, Amini A. Shifting paradigms: whole brain radiation therapy versus stereotactic radiosurgery for brain metastases. CNS Oncol. 2019;8(1):CNS27.

16. Yamamoto M, Kawabe T, Sato Y, Higuchi Y, Nariai T, Watanabe S, et al. Stereotactic radiosurgery for patients with multiple brain metastases: a case-matched study comparing treatment results for patients with 2-9 versus 10 or more tumors. J Neurosurg. 2014;121 Suppl:16-25.

17. Brown PD, Ballman KV, Cerhan JH, Anderson SK, Carrero XW, Whitton AC, et al. Postoperative stereotactic radiosurgery compared with whole brain radiotherapy for resected metastatic brain disease (NCCTG N107C/CEC.3): a multicentre, randomised, controlled, phase 3 trial. Lancet Oncol. 2017;18(8):1049-60.

18. Kim DH, Schultheiss TE, Radany EH, Badie B, Pezner RD. Clinical outcomes of patients treated with a second course of stereotactic radiosurgery for locally or regionally recurrent brain metastases after prior stereotactic radiosurgery. J Neurooncol. 2013;115(1):37-43.

19. Shultz DB, Modlin LA, Jayachandran P, Von Eyben R, Gibbs IC, Choi CYH, et al. Repeat Courses of Stereotactic Radiosurgery (SRS), Deferring Whole-Brain Irradiation, for New Brain Metastases After Initial SRS. Int J Radiat Oncol Biol Phys. 2015;92(5):993-9.

20. Brown PD, Jaeckle K, Ballman KV, Farace E, Cerhan JH, Anderson SK, et al. Effect of Radiosurgery Alone vs Radiosurgery With Whole Brain Radiation Therapy on Cognitive Function in Patients With 1 to 3 Brain Metastases: A Randomized Clinical Trial. JAMA. 2016;316(4):401-9.

21. Mehta MP, Kotecha R. Postoperative Management of Resected Brain Metastases: When Can Radiotherapy Be Deferred? J Clin Oncol. 2018:JC02018793232. 
22. Wolf A, Kvint S, Chachoua A, Pavlick A, Wilson M, Donahue B, et al. Toward the complete control of brain metastases using surveillance screening and stereotactic radiosurgery. J Neurosurg. 2018;128(1):23-31.

23. Aiyama H, Yamamoto M, Kawabe T, Watanabe S, Koiso T, Sato Y, et al. Complications after stereotactic radiosurgery for brain metastases: Incidences, correlating factors, treatments and outcomes. Radiother Oncol. 2018;129(2):364-9.

24. Rosell R, Carcereny E, Gervais R, Vergnenegre A, Massuti B, Felip E, et al. Erlotinib versus standard chemotherapy as first-line treatment for European patients with advanced EGFR mutation-positive non-small-cell lung cancer (EURTAC): a multicentre, open-label, randomised phase 3 trial. Lancet Oncol. 2012;13(3):239-46.

25. Lu F, Hou Y, Xia Y, Li L, Wang L, Cao K, et al. Survival and intracranial control outcomes of wholebrain radiotherapy (WBRT) alone versus WBRT plus a radiotherapy boost in non-small-cell lung cancer with brain metastases: a single-institution retrospective analysis. Cancer Manag Res. 2019;11:4255-72.

26. Nagtegaal SHJ, Claes A, Suijkerbuijk KPM, Schramel F, Snijders TJ, Verhoeff JJC. Comparing survival predicted by the diagnosis-specific Graded Prognostic Assessment (DS-GPA) to actual survival in patients with 1-10 brain metastases treated with stereotactic radiosurgery. Radiother Oncol. 2019;138:173-9.

27. Woody NM, Greer MD, Reddy CA, Videtic GMM, Chao ST, Murphy ES, et al. Validation of the DiseaseSpecific GPA for Patients With 1 to 3 Synchronous Brain Metastases in Newly Diagnosed NSCLC. Clin Lung Cancer. 2018;19(1):e141-e7.

28. Sung S, Lee SW, Kwak YK, Kang JH, Hong SH, Kim YS. Intracranial control and survival outcome of tyrosine kinase inhibitor (TKI) alone versus TKI plus radiotherapy for brain metastasis of epidermal growth factor receptor-mutant non-small cell lung cancer. J Neurooncol. 2018;139(1):205-13.

29. Welsh JW, Komaki R, Amini A, Munsell MF, Unger W, Allen PK, et al. Phase II trial of erlotinib plus concurrent whole-brain radiation therapy for patients with brain metastases from non-small-cell lung cancer. J Clin Oncol. 2013;31(7):895-902.

30. Chang WY, Wu YL, Su PL, Yang SC, Lin CC, Su WC. The impact of EGFR mutations on the incidence and survival of stages I to III NSCLC patients with subsequent brain metastasis. PLoS One. 2018;13(2):e0192161.

31. Ge M, Zhuang Y, Zhou X, Huang R, Liang X, Zhan Q. High probability and frequency of EGFR mutations in non-small cell lung cancer with brain metastases. J Neurooncol. 2017;135(2):413-8.

32. Khalifa J, Amini A, Popat S, Gaspar LE, Faivre-Finn C, International Association for the Study of Lung Cancer Advanced Radiation Technology C. Brain Metastases from NSCLC: Radiation Therapy in the Era of Targeted Therapies. J Thorac Oncol. 2016;11(10):1627-43.

33. Magnuson WJ, Lester-Coll NH, Wu AJ, Yang TJ, Lockney NA, Gerber NK, et al. Management of Brain Metastases in Tyrosine Kinase Inhibitor-Naive Epidermal Growth Factor Receptor-Mutant Non-SmallCell Lung Cancer: A Retrospective Multi-Institutional Analysis. J Clin Oncol. 2017;35(10):1070-7. 
34. Yang JJ, Zhou C, Huang Y, Feng J, Lu S, Song Y, et al. Icotinib versus whole-brain irradiation in patients with EGFR-mutant non-small-cell lung cancer and multiple brain metastases (BRAIN): a multicentre, phase 3, open-label, parallel, randomised controlled trial. Lancet Respir Med. 2017;5(9):707-16.

35. Bebb DG, Agulnik J, Albadine R, Banerji S, Bigras G, Butts C, et al. Crizotinib inhibition of ROS1positive tumours in advanced non-small-cell lung cancer: a Canadian perspective. Curr Oncol. 2019;26(4):e551-e7.

36. Liu C, Yu H, Long Q, Chen H, Li Y, Zhao W, et al. Real World Experience of Crizotinib in 104 Patients With ALK Rearrangement Non-small-cell Lung Cancer in a Single Chinese Cancer Center. Front Oncol. 2019;9:1116.

37. Yang Y, Zhou J, Zhou J, Feng J, Zhuang W, Chen J, et al. Efficacy, safety, and biomarker analysis of ensartinib in crizotinib-resistant, ALK-positive non-small-cell lung cancer: a multicentre, phase 2 trial. Lancet Respir Med. 2020;8(1):45-53.

38. Buttigliero C, Bertaglia V, Novello S. Anti-angiogenetic therapies for central nervous system metastases from non-small cell lung cancer. Transl Lung Cancer Res. 2016;5(6):610-27.

39. Besse B, Le Moulec S, Mazieres J, Senellart H, Barlesi F, Chouaid C, et al. Bevacizumab in Patients with Nonsquamous Non-Small Cell Lung Cancer and Asymptomatic, Untreated Brain Metastases (BRAIN): A Nonrandomized, Phase II Study. Clin Cancer Res. 2015;21(8):1896-903.

40. Yamamoto M, Serizawa T, Shuto T, Akabane A, Higuchi Y, Kawagishi J, et al. Stereotactic radiosurgery for patients with multiple brain metastases (JLGK0901): a multi-institutional prospective observational study. Lancet Oncol. 2014;15(4):387-95.

\section{Table}

\section{Table 1 Patient baseline characteristics}




\begin{tabular}{|l|l|ll|}
\hline Characteristics & & \multicolumn{2}{|c|}{ No.of patients[\% } \\
\hline Sex & Female & 36 & $(45.0 \square$ \\
& Male & 44 & $(55.0)$ \\
\hline Age,years & $\leq 60$ & 56 & $(70.0)$ \\
& $\square 60$ & 24 & $(30.0)$ \\
\hline KPS scores & $\leq 70$ & 12 & $(15.0)$ \\
& $\square 70$ & 68 & $(85.0)$ \\
\hline The first diagnosis is BM & No & 51 & $(63.8)$ \\
& Yes & 29 & $(36.3)$ \\
\hline NSCLC-BMs,months & $\leq 6$ & 42 & $(52.5)$ \\
& $\square 6$ & 38 & $(47.5)$ \\
\hline Pathological & Adenocarcinoma & 63 & $(78.8)$ \\
& Other & 17 & $(21.3)$ \\
\hline Neurological symptom & Yes & 34 & $(42.5)$ \\
& No & 46 & $(57.5)$ \\
\hline Location of BMs & Epencephalon & 4 & $(5.0 \square$ \\
& Cerebrum & 62 & $(77.5)$ \\
& Epencephalon+Cerebrum & 14 & $(17.5)$ \\
\hline Number of BMs & $\leq 3$ & 65 & $(81.3)$ \\
& $\square 3$ & 15 & $(18.8)$ \\
\hline BM size,cm & $\leq 3$ & 68 & $(85.0)$ \\
& $\square 3$ & 12 & $(15.0)$ \\
\hline EMs & No & 42 & $(52.5)$ \\
& Yes & 38 & $(47.5)$ \\
\hline Number of organs with EMs & 0.0 & 42 & $(52.5)$ \\
& 1.0 & 16 & $(20.0)$ \\
& $\geq 2$ & 22 & $(27.5)$ \\
\hline Controlled of primary & No & 23 & $(28.8)$ \\
cancer and EMs & Yes & 57 & $(71.3)$ \\
\hline GPA scores & $0-2$ & 24 & $(30.0)$ \\
& $2.5-3$ & 34 & $(42.5 \square$ \\
& $3.5-4$ & 21 & $(26.3 \square$ \\
\hline RPA class & 1 & 31 & $(38.8)$ \\
& II & 36 & $(57.5)$ \\
& III & & \\
& & $3.8)$ \\
\hline
\end{tabular}

(Continued)

Table 1 (Continued). 


\begin{tabular}{|l|l|ll|}
\hline Characteristics & & No.of patients[\%] \\
\hline Genetic mutations & EGFR positive & 16 & $(20.0)$ \\
& Other genes positive & 6 & $(7.5 \square$ \\
& Wild type & 32 & $(40.0)$ \\
& Unknown & 26 & $(32.5)$ \\
\hline Other treatments & No & 34 & $(42.5)$ \\
& Yes & 46 & $(57.5)$ \\
\hline Other treatments & Supportived therapy & 34 & $(42.5)$ \\
& Chemotherapy & 18 & $(22.5)$ \\
& Targeted therapy & 16 & $(20.0)$ \\
& Chemotherapy+Targeted & 12 & $(15.0)$ \\
\hline Targeted therapy with HSRT & EGFR-TKI & 14 & $(17.5)$ \\
& ALK/ROS1-TKI & 5 & $(6.3)$ \\
& VEGFR & 9 & $(11.3)$ \\
\hline HSRT technology & TOMO & 37 & $(46.3)$ \\
& VMAT & 43 & $(53.7)$ \\
\hline Dose of HSRT,Gy & PTV 30 30G/3f & 5 & $(6.2)$ \\
& PTV=30Gy/3f & 51 & $(63.8)$ \\
& PTV[30Gy/3f & 24 & $(30.0)$ \\
\hline Progress to the end point & No & 48 & $(60.0)$ \\
& Yes & 31 & $(38.8)$ \\
\hline
\end{tabular}

Abbreviations: KPS, Karnofsky Performance Scale; BMs, brain metastases; NSCLC BMs, the time interval from cancer diagnosis to confifirmed BMs; EMs, extracranial metastases; GPA, graded prognostic assessment; RPA, recursive partitioning analysis; EGFR, epidermal growth factor receptor; ALK, anaplastic lymphoma kinase; ROS 1, c-ros oncogene 1 receptor kinase; VEGFR, anti-vascular endothelial growth factor; TKIs, tyrosine kinase inhibitors; HSRT, hypofractionated stereotactic radiotherapy; TOMO, helical tomotherapy; VMAT, volumetric modulated arc therapy; PTV, planning target volume.

\section{Figures}




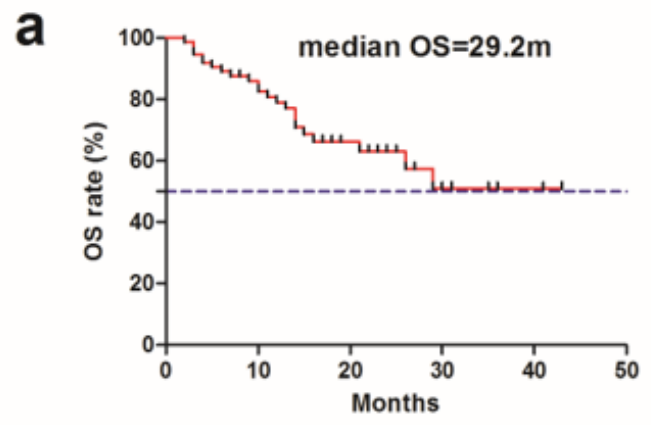

$\begin{array}{cccc}6 \text { months } & 12 \text { months } & 24 \text { months } & 36 \text { months } \\ 89.1 \% & 79.0 \% & 63.1 \% & 51.0 \%\end{array}$
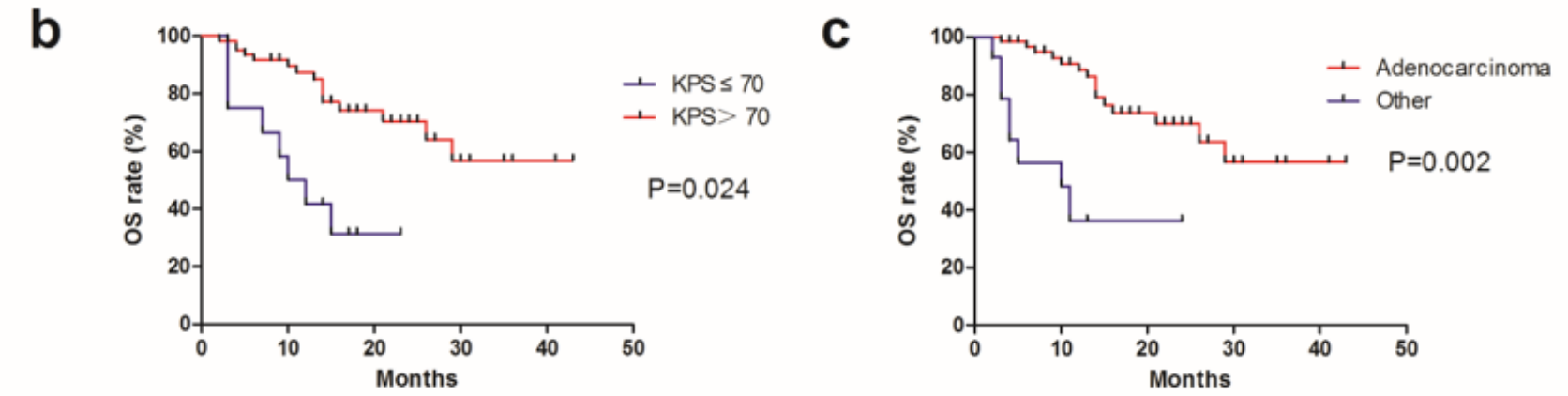

12 months

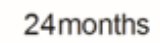

36 months

median OS

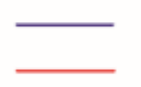

$41.7 \%$

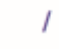

$56.9 \%$

$12.7 \mathrm{~m}$

$31.7 \mathrm{~m} \longrightarrow 36.2 \%$

12 months

24 months

36 months

median OS

$87.4 \%$

$70.4 \%$

$70.0 \%$

$56.6 \%$

$31.9 \mathrm{~m}$

$36.2 \%$

I

$12.4 \mathrm{~m}$

d

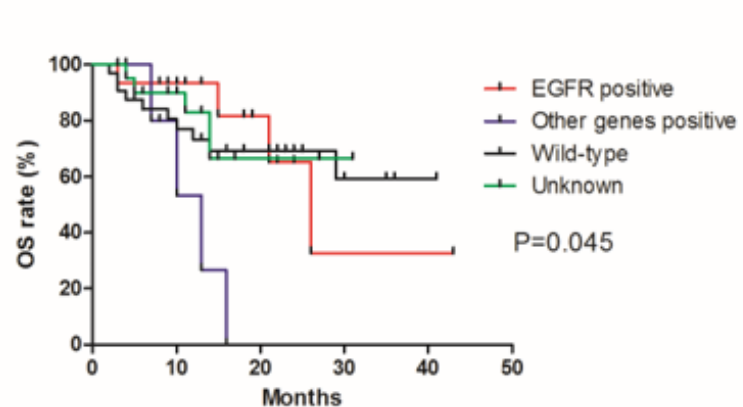

e

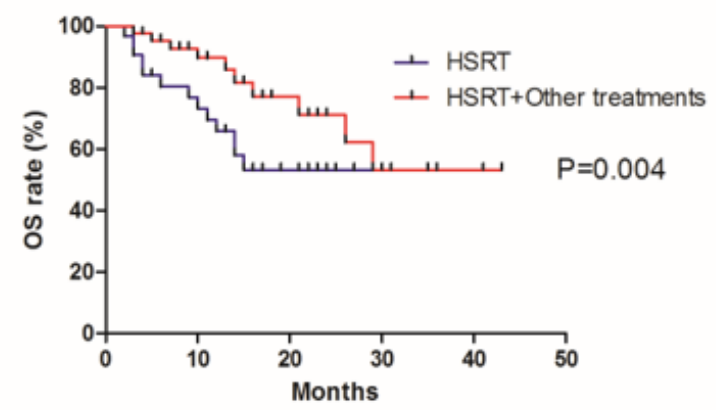

$\begin{array}{cccc}\text { 12months } & \text { 24months } & 36 \text { months } & \text { median OS } \\ 93.3 \% & 65.3 \% & 32.7 \% & 27.9 \mathrm{~m} \\ 53.3 \% & 0.0 \% & & 11.8 \mathrm{~m} \\ 73.3 \% & 69.2 \% & 59.3 \% & 29.5 \mathrm{~m} \\ 83.0 \% & 66.4 \% & / & 24.1 \mathrm{~m}\end{array}$

12 months 24 months 36 months median OS

$\begin{array}{llll}65.9 \% & 53.3 \% & \text { / } & 22.7 \mathrm{~m}\end{array}$

$89.7 \%$

$71.2 \%$

$53.4 \%$

$31.6 \mathrm{~m}$

\section{Figure 1}

Cumulative incidence of OS for all patients (a); comparison of cumulative incidence of OS between KPS $\leq 70$ group and KPS $\otimes 70$ group (b), Adenocarcinoma group and non-adenocarcinoma group (c), EGFR positive group and other genes mutations (d), HSRT alone group and HSRT combined other treatments group (e). Abbreviations: OS, overall survival; KPS, Karnofsky Performance Scale; EGFR, epidermal growth factor receptor; HSRT, hypofractionated stereotactic radiotherapy. 
a

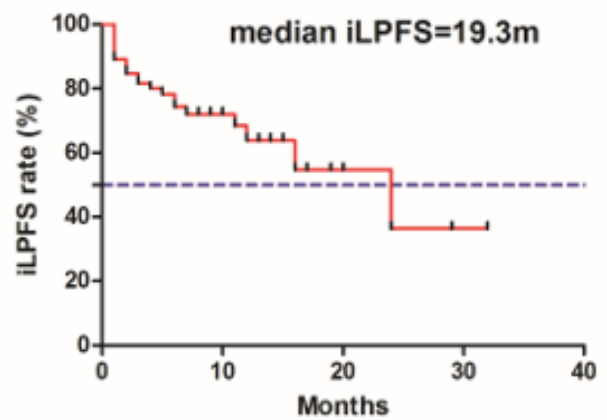

6months

$78.3 \%$

12 months

$63.9 \%$

24months

$36.5 \%$
C

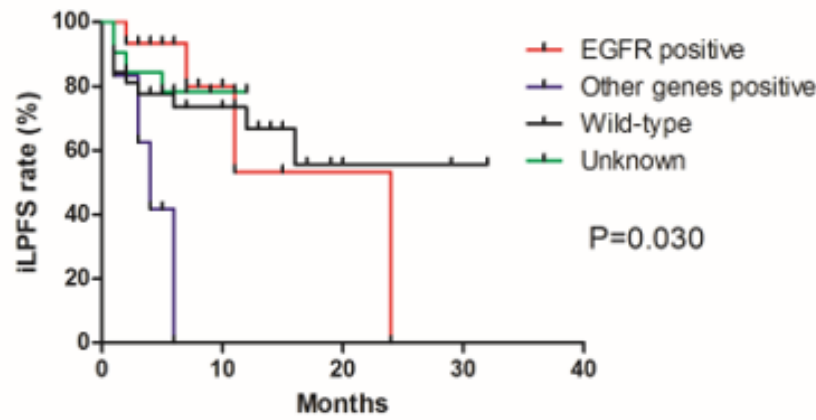

12 months

24months

30 months

median iLPFS

$53.3 \%$

$0.0 \%$

$0.0 \%$

$66.8 \%$

$78.4 \%$
$55.6 \%$

I
30 months

$36.5 \%$

$16.8 \mathrm{~m}$

$4.1 \mathrm{~m}$

$55.6 \%$
$9.9 \mathrm{~m}$ b

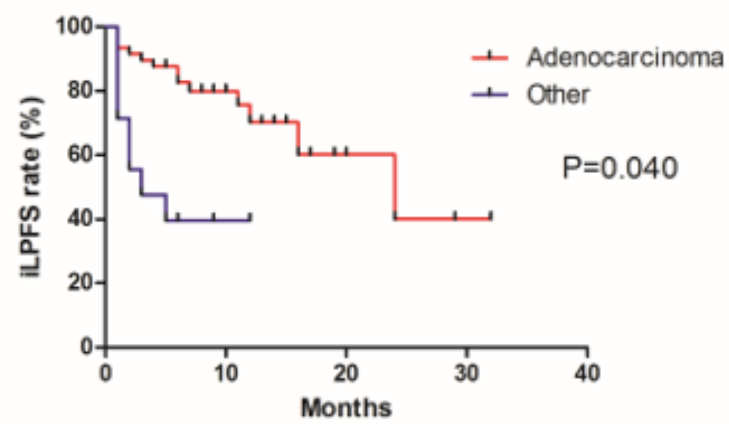

12months 24 months 30 months median iLPFS

$70.2 \%$

$40.1 \%$

$40.1 \%$

$21.1 \mathrm{~m}$

$39.7 \%$

I

$6.0 \mathrm{~m}$

d

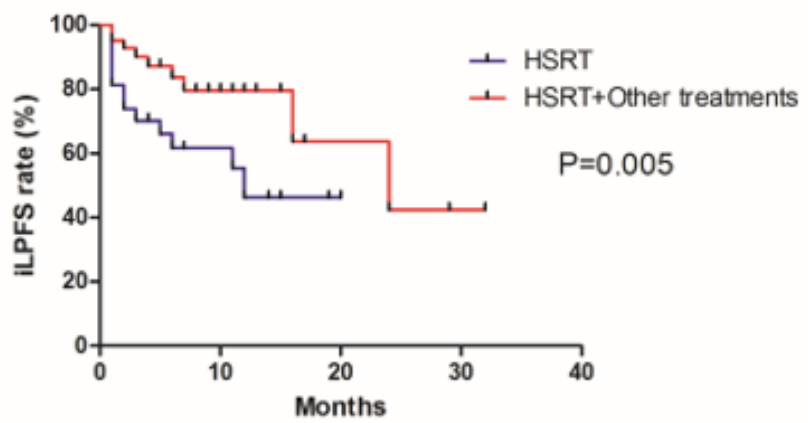

12months 24 months 30 months median iLPFS

$46.2 \%$

$79.6 \%$

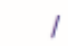

$42.5 \%$
$12.0 \mathrm{~m}$

$22.0 \mathrm{~m}$

\section{Figure 2}

Cumulative incidence of iLPFS for all patients (a); comparison of cumulative incidence of iLPFS between Adenocarcinoma group and non-adenocarcinoma group (b), EGFR positive group and other genes mutations (c), HSRT alone group and HSRT combined other treatments group (d). Abbreviations: iLPFS, intracranial local progression-free survival; EGFR, epidermal growth factor receptor; HSRT, hypofractionated stereotactic radiotherapy. 
a

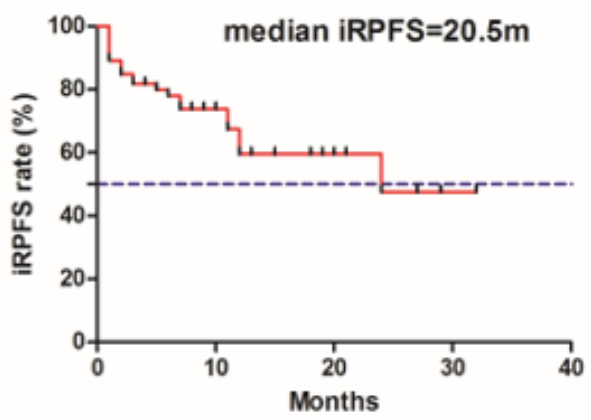

6months 12 months 24 months 30 months

$78.0 \%$

$67.4 \%$

$47.5 \%$

$47.5 \%$

C

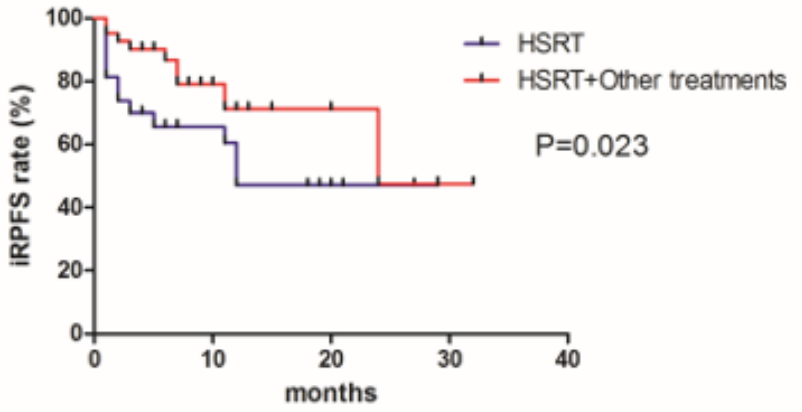

12 months

24 months

30 months

$47.1 \%$

$47.5 \%$ median IRPFS

$16.5 \mathrm{~m}$

$22.7 \mathrm{~m}$ b

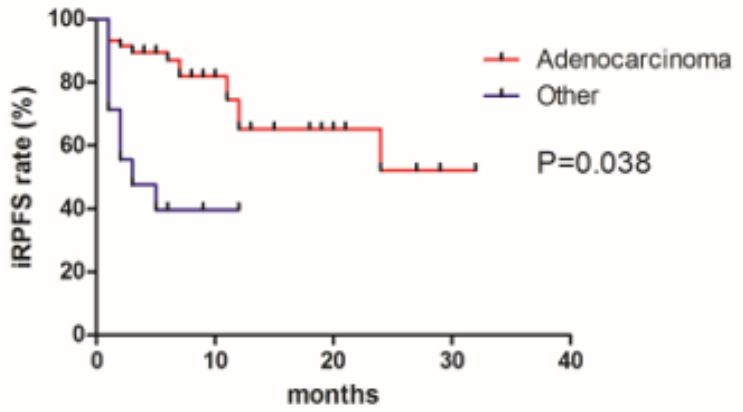

12 months 24 months 30months median iRPFS

$74.6 \%$

$52.2 \%$

$52.2 \%$

$22.5 \mathrm{~m}$

$6.0 \mathrm{~m}$

d

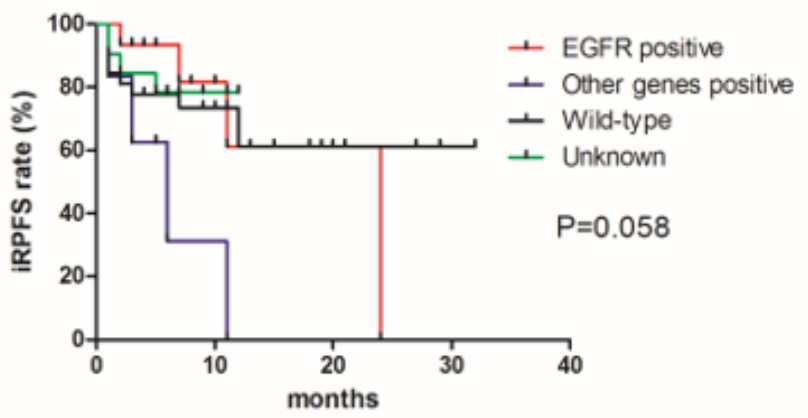

12 months

24months

30months

median IRPFS

$61.3 \%$

$0.0 \%$

$17.9 \mathrm{~m}$

$0.0 \%$

$6.1 \mathrm{~m}$

$61.2 \%$

$61.2 \%$

$21.7 \mathrm{~m}$

$9.9 \mathrm{~m}$

\section{Figure 3}

Cumulative incidence of iRPFS for all patients (a); comparison of cumulative incidence of iRPFS between Adenocarcinoma group and non-adenocarcinoma group (b), HSRT alone group and HSRT combined other treatments group (c), EGFR positive group and other genes mutations (d). Abbreviations: iRPFS, intracranial regional progression-free survival; HSRT, hypofractionated stereotactic radiotherapy; EGFR, epidermal growth factor receptor. 

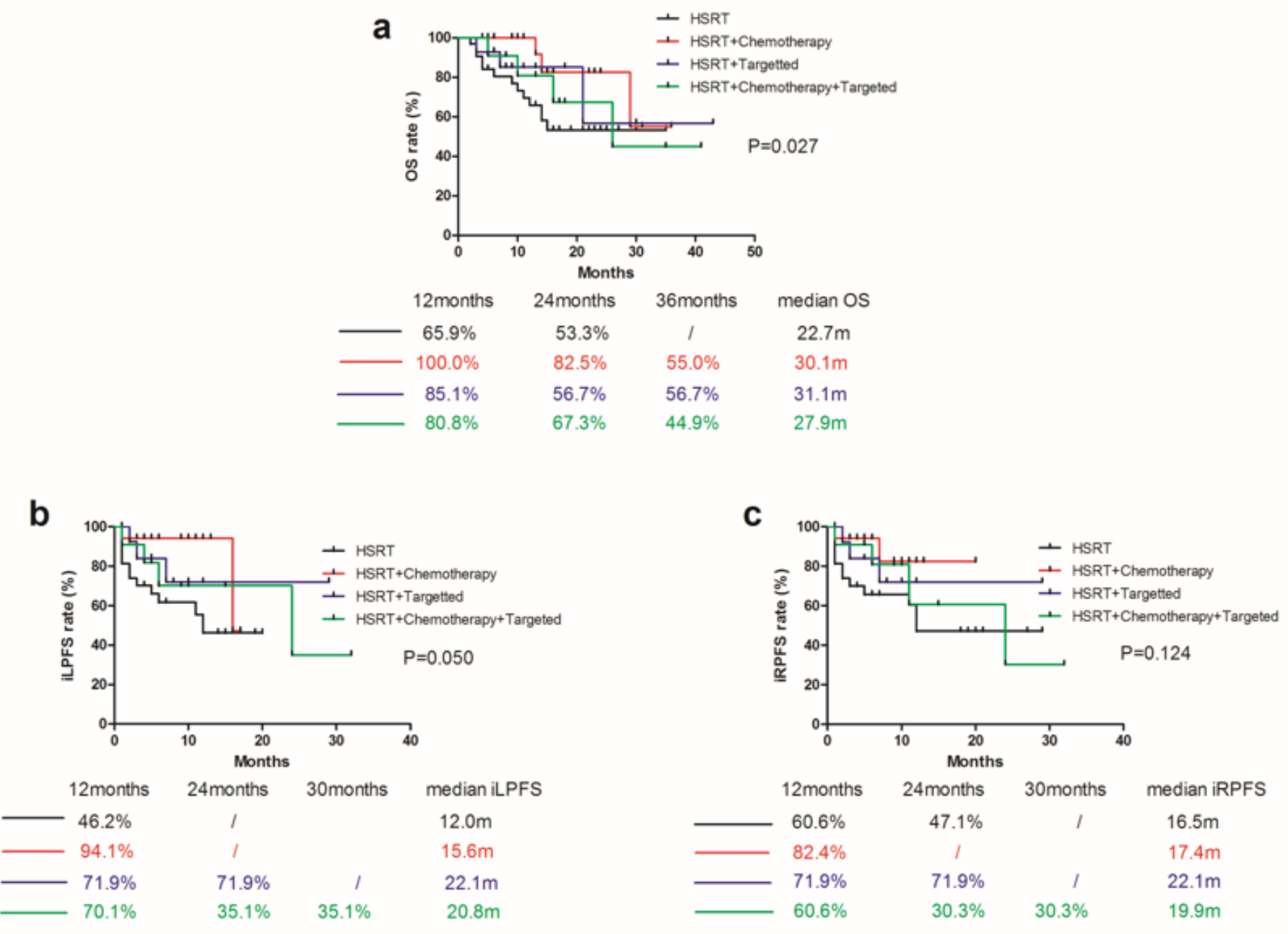

\section{Figure 4}

Comparison of cumulative incidence of OS (a), iLPFS (b), and iRPFS (c) between HSRT alone group and HSRT combined other treatments group (chemotherapy, targeted therapy,chemotherapy and targeted therapy). Abbreviations: OS, overall survival; iLPFS, intracranial local progression-free survival; iRPFS, intracranial regional progression-free survival; HSRT, hypofractionated stereotactic radiotherapy.

\section{Supplementary Files}

This is a list of supplementary files associated with this preprint. Click to download.

- Supplementary.docx 